\title{
COMPÓSITO MAGNÉTICO DE AMIDO MODIFICADO APLICADO À CONCENTRAÇÃO DE REJEITOS DE MINERAÇÃO VISANDO INCREMENTO NAS RECUPERAÇÕES DE HEMATITA $\left(\mathrm{Fe}_{2} \mathrm{O}_{3}\right)$
}

\author{
Daniel R. S. Nogueira, ${ }^{1}$ Mario José Politi ${ }^{1}$ \\ 1 -Instituto de Química, Universidade de São Paulo (USP), São Paulo, SP \\ drsn_aniel@usp.br
}

\begin{abstract}
Resumo: A geração e disposição de resíduos são fatores críticos na mineração, com importantes implicações econômicas, ambientais e sociais. A necessidade de dar destinação sustentável aos resíduos da mineração, em especial os rejeitos, ganhou mais relevância após os desastres das barragens de rejeito em Mariana (2015) e Brumadinho (2019), e o aproveitamento econômico desses materiais surge como alternativa à deposição em estruturas como pilhas e barragens. Com base nos dados declarados no Relatório Anual de Lavra (RAL), no período de 2010 a 2019, estima-se que, no Brasil, um total de 3,4 bilhões de toneladas de rejeitos e 8,2 bilhões de toneladas de estéril foram geradas em empreendimentos produtores de ferro, ouro, cobre, fosfato, estanho, alumínio, níquel, carvão, manganês, zinco, cromo e vanádio. Atualmente, para elevação de teores, o processo de concentração mineral mais utilizado é por flotação, processo no qual as propriedades de superfície dos materiais são induzidas à hidrofobicidade por meio de produtos químicos chamados de agentes coletores. Como estratégia à redução da geração de rejeitos verificamos o uso de um compósito magnético de amido modificado aplicado às partículas minerais de forma a induzirmos o magnetismo ambiental das partículas de modo seletivo à hematita, conferindo-lhes maior susceptibilidade magnética durante a concentração magnética de alto campo.
\end{abstract}

Palavras-chave: economia circular, concentração magnética de alto campo a úmido, amido modificado.

\section{Modified starch magnetic composite applied to concentration of tailings for increasing recoveries of hematite $\left(\mathrm{Fe}_{2} \mathrm{O}_{3}\right)$}

\begin{abstract}
Waste generation and disposal are critical factors in mining, with important economic, environmental, and social implications. The need to provide sustainable disposal of mining waste, especially tailings, has gained more relevance after the tailings dam disasters in Mariana (2015) and Brumadinho (2019), and the economic use of these materials emerges as an alternative to disposal in structures such as piles and dams. Based on data declared in the Annual Mining Report (RAL), in the period from 2010 to 2019, it is estimated that, in Brazil, a total of 3.4 billion tons of tailings and 8.2 billion tons of waste rock were generated in enterprises producing iron, gold, copper, phosphate, tin, aluminum, nickel, coal, manganese, zinc, chromium and vanadium. Currently, the most used mineral concentration process is froth flotation, a process in which the surface properties of materials are induced to hydrophobicity by means of chemicals called collecting agents. As a strategy to reduce the generation of tailings, we verified the use of a modified starch magnetic composite applied to mineral particles in order to selectively induce the environmental magnetism of the particles to hematite, giving them greater magnetic susceptibility during high-field magnetic concentration.
\end{abstract}

Keywords: circular economy, wet high intensity magnetic separator, modified starch.

\section{Introdução}

Segundo Chaves (2006) a flotação é o método de concentração mais largamente adotado para a faixa granulométrica fina $(<150 \mu \mathrm{m})$. A concentração de minérios por flotação apresenta boa eficiência em uma dada faixa de tamanho de partícula, fora da qual a recuperação de finos ou grossos é muito baixa. Essa faixa de tamanho depende da espécie mineral, da escala de operação e da concentração de reagentes, e flutua para os minérios de $5 \mu \mathrm{m}$ e $150 \mu \mathrm{m}$. Em razão da baixa recuperação, principalmente nas frações finas e grossas, milhares de toneladas de rejeitos com altos teores têm sido depositados em barragens. Um evento na mineração brasileira, também conhecido 
como desastre de Mariana, ocorreu em 5 de novembro de 2015 na barragem do Fundão localizada em ambiente de mina na Cidade de Mariana, Estado de Minas Gerais. O rompimento teve 19 vítimas fatais e provocou a vazão de 32,6 milhões de metros cúbicos de rejeitos da estrutura. Após 3 anos da tragédia de Mariana, no dia 25 de janeiro de 2019, outra barragem de mineração composta de rejeitos de minério de ferro rompeu-se catastroficamente na Cidade de Brumadinho, Minas Gerais, Brasil. A ruptura produziu uma avalanche de lama que se espalhou por $10 \mathrm{~km}$ e atingiu o Rio Paraopeba; um importante afluente do rio São Francisco. Apesar do volume de lama derivado do desastre de Brumadinho ser menor do que o de Mariana, o evento causou perdas muito mais significativas de vidas. Tendo em vista o impacto desses tipos de acidente, estudos têm sido realizados para o beneficiamento de rejeitos ou ROM (run of mine) induzindo a superfície dos materiais à hidrofobicidade por meio agentes coletores aplicados no processo de concentração por flotação. Entretanto, quando o beneficiamento é realizado pelo processo de concentração magnética depende principalmente da susceptibilidade magnética e do magnetismo ambiental das partículas minerais. Dentro desse contexto, o presente trabalho tem a intenção de contribuir com opções de reaproveitamento de rejeitos e/ou melhorias no ROM, e para isso propôs o uso de um compósito magnético de amido modificado aplicado na rota de concentração magnética como forma de induzir seletivamente o magnetismo das partículas minerais e justificar o seu beneficiamento ou melhoria do processo.

\section{Experimental}

Amostras de rejeitos de mineração

$500 \mathrm{~g}$ de rejeito de zinco, com umidade de $10 \%$, foram direcionadas para análises de fluorescência e difração de raios-x (modelo Zetim - Malvern Panalytical). Para análise de tamanho de partículas utilizamos espalhamento a baixo ângulo de luz laser (LALLS - Mastersizer 2000 - Malvern Panalytical).

\section{Gelatinização química do amido de milho modificado de fonte regular}

Em um béquer foi pesado $50 \mathrm{~g} / \mathrm{L}$ de amido modificado (base seca), em outro béquer foi pesado água destilada para uma solução à $5 \%(\mathrm{p} / \mathrm{v})$, adicionou-se essa água destilada ao amido formando uma pasta, em um terceiro béquer, foi pesado $8 \mathrm{~g} / \mathrm{L}$ de uma solução de soda caustica em uma concentração de 50\%. Por meio de um agitador mecânico ajustamos a velocidade em $1200 \mathrm{rpm}$ e iniciamos a adição da solução de soda caustica (50\%) à solução de pasta de amido, por fim ajustamos o teor de sólidos dessa solução final à $3 \%(\mathrm{p} / \mathrm{v})$ utilizando água destilada e agitamos por $10 \mathrm{~min}$, como controle ao processo foi medido e registrar o valor de $\mathrm{pH}$.

Sintese de partículas magnéticas via coprecipitação

Uma solução de precursores $\mathrm{Fe}^{3+} / \mathrm{Fe}^{2+}$ 0,2/0,1 mol/L, onde $\mathrm{Fe}^{3+}$ (cloreto férrico hexahidratado $\mathrm{FeCl}_{3} .6 \mathrm{H}_{2} \mathrm{O}$-Sigma Aldrich) e $\mathrm{Fe}^{2+}$ (Cloreto ferroso tetrahidratado $\mathrm{FeCl}_{2} \cdot 4 \mathrm{H}_{2} \mathrm{O}-$ Sigma Aldrich) permaneceu em agitação (1000rpm) durante $10 \mathrm{~min}$. Em seguida, uma solução de soda caustica a uma concentração de $50 \%(\mathrm{v} / \mathrm{v})$ foi adicionada a esses precursores por meio de uma bomba dosadora $(5 \mathrm{ml} / \mathrm{min})$, por fim aquecemos o sistema a $70{ }^{\circ} \mathrm{C}$, por $20 \mathrm{~min}$, para promover a coprecipitação das partículas magnéticas (Eq.1).

$$
\mathrm{Fe}^{2+}+2 \mathrm{Fe}^{3+}+8 \mathrm{OH}^{-} \rightarrow \mathrm{Fe}_{3} \mathrm{O}_{4}+4 \mathrm{H}_{2} \mathrm{O}
$$


Incorporação do amido gelatinizado nas partículas magnéticas

As partículas magnéticas foram adicionadas no amido gelatinizado $5 \mathrm{~g} / \mathrm{L}$ e agitadas a $100 \mathrm{rpm}$. por 30 min com o objetivo de incorporar essas partículas na matriz polimérica de amido modificado.

\section{Resultados e discussão}

Amostras de rejeitos de zinco analisadas por difração de raio laser (LALLS) estão apresentadas na figura 1.

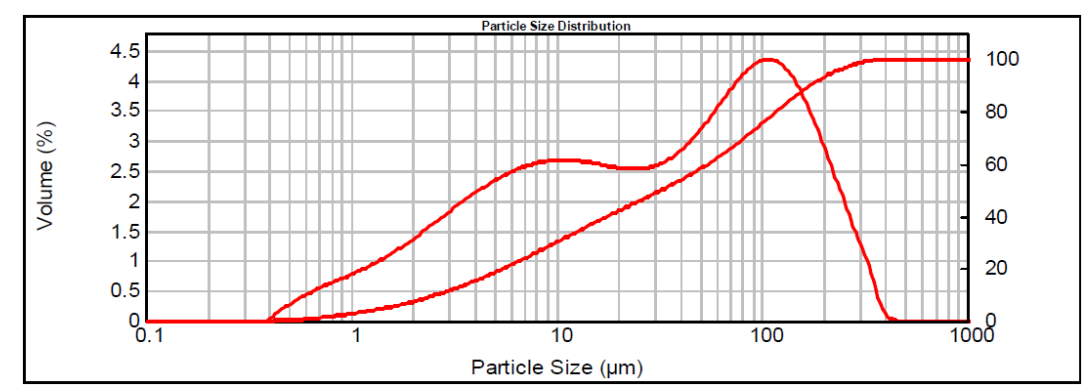

Figura 1: Distribuição granulométrica por difração de raio laser (LALLS)

Observa-se na figura 1 a uma distribuição granulométrica abaixo de 178,25 mm (size $\mu \mathrm{m})$ temos cerca de $90 \%$ de volume da amostra. A figura 2, apresenta os resultados da difração de raio-X (analisador XRD) também para os rejeitos de zinco, onde observa-se a presença da fase hematita $\left(\mathrm{Fe}_{2} \mathrm{O}_{3}\right)$.

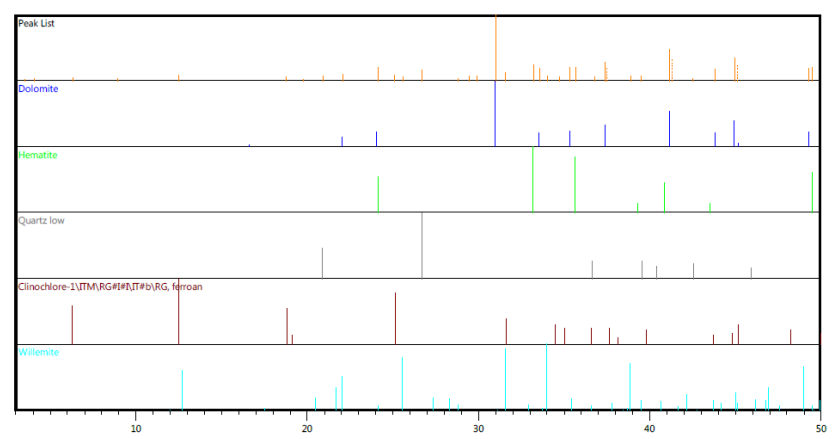

Figura 2: Identificação de fases por difração de raios-X (analisador XRD) dos rejeitos de zinco

A tabela 1 exibe a distribuição modal dos minerais contidos na amostra de rejeitos de zinco em porcentagem por elemento químico, os teores apresentados foram dosados em amostra prensada, na calibração STD-1 (Standardless) relativa à análise sem padrões dos elementos químicos compreendidos entre o flúor de urânio. A perda ao fogo (PF) foi realizada a $1020^{\circ} \mathrm{C}$ por $2 \mathrm{~h}$.

$\begin{array}{lllllllllllllllllllll}\mathrm{Na}_{2} \mathrm{O} & \mathrm{MgO}_{\mathrm{g}} & \mathrm{Al}_{2} \mathrm{O}_{3} & \mathrm{SiO}_{2} & \mathrm{P}_{2} \mathrm{O}_{5} & \mathrm{SO}_{3} & \mathrm{Cl} & \mathrm{K}_{2} \mathrm{O} & \mathrm{CaO}_{\mathrm{aO}} & \mathrm{TiO}_{2} & \mathrm{~V}_{2} \mathrm{O}_{5} & \mathrm{Cr}_{2} \mathrm{O}_{3} & \mathrm{MnO} & \mathrm{Fe}_{2} \mathrm{O}_{3} & \mathrm{NiO} & 2 \mathrm{nO} & \mathrm{SrO} & \mathrm{BaO} & \mathrm{PbO} & \mathrm{PF}\end{array}$

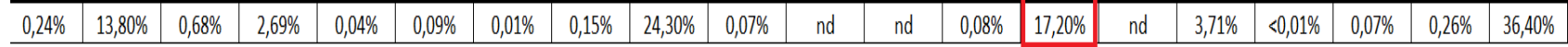

Tabela 1: Análise química por fluorescência de raios-X (XRF) 
Observamos através da tabela 1 que o teor do contaminante hematita $\left(\mathrm{Fe}_{2} \mathrm{O}_{3}\right)$ analisado está presente na forma elementar em 17,20\% da amostra de rejeitos de zinco. Na figura 3 temos o WHC01B um concentrador magnético do tipo "Wet High Intensity Magnetic Separator”. O princípio de funcionamento deste equipamento consiste na circulação de corrente elétrica induzida por entre os polos das bobinas resultando em um alto gradiente e intensidade de campo eletromagnético na zona de separação. O equipamento utilizado para os ensaios é composto principalmente por bobina geradora de campo magnético $(8000,10000$ e 13800 Gauss) e matrizes do tipo placa ranhurada $(1,5$ e $2,5 \mathrm{~mm}$ ). Este equipamento está localizado no laboratório de desenvolvimento do fabricante Inbrás-Eriez.

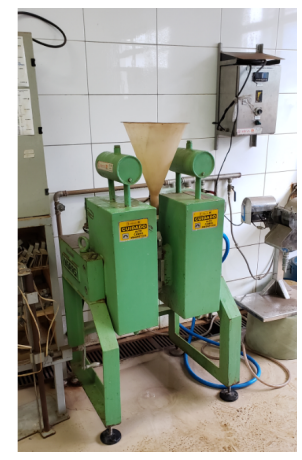

Figura 3 - Wet High Intensity Magnetic Separator (WHIMS)

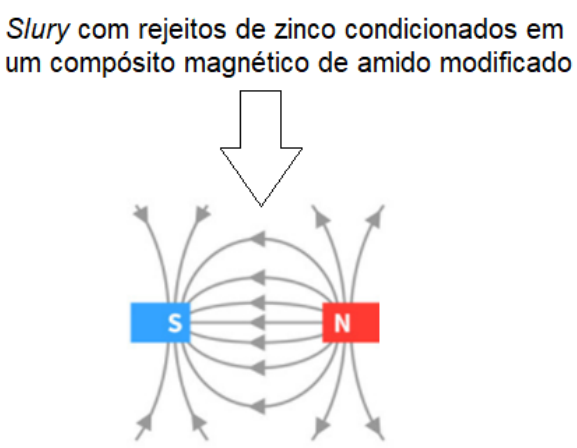

Figura 4 - Esquema simplificado da concentração magnética de rejeitos de zinco e compósito magnético de amido modificado

Conforme ilustrado na figura 4, foi adicionada a solução do compósito magnético de amido modificado ao "slurry" ou polpa (minério e água), condicionados por 2 minutos e direcionados à alimentação do concentrador magnético de alto campo. Ao entrarem na zona de separação, uma certa quantidade de massa chamada de concentrado magnético é atraída pela superfície da matriz. Sob ação da gravidade combinada com a hidrodinâmica do sistema o restante da massa desse "slurry" é descartado do processo como rejeito não-magnético. Em seguida o material é filtrado sob pressão, seco em estufa a $110^{\circ} \mathrm{C}$, desagregado com auxílio de peneiras, pesado, embalado e destinado às análises de fluorescência de raio-X e espectrometria de absorção atômica para elementos que contém uma faixa de detecção mais baixa (por exemplo o Cádmio).

Os ensaios de concentração magnética foram planejados na etapa "rougher", ou seja, um estágio inicial de qualquer operação de tratamento de minério onde são produzidos concentrados e rejeito de teores supostamente ainda inaceitáveis e que por isso, a depender, precisam ser reprocessados respectivamente nos estágios posteriores chamados de "cleaner" e "scavenger". As etapas estão ilustradas abaixo na figura 5, onde classificamos como alimentação nova os rejeitos de zinco.

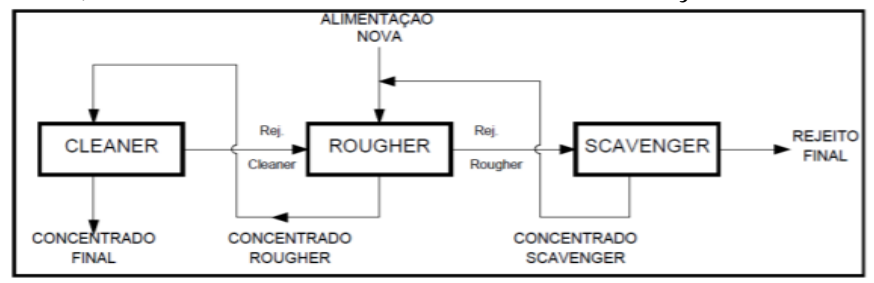

Figura 5 - Esquema representativo das etapas de concentração magnética 
Os resultados dos ensaios de concentração magnética utilizando os rejeitos de zinco (alimentação nova) estão relacionados abaixo na tabela 2.

\begin{tabular}{|l|l|c|c|c|}
\hline \multirow{2}{*}{ Etapa } & Material & \multicolumn{2}{|c|}{$\begin{array}{r}\text { Variação da hematita }\left(\mathrm{Fe}_{2} \mathrm{O}_{3}\right) \text { com uso de um compósito magnético de } \\
\text { amido modificado em relação a amostra em branco }\end{array}$} \\
\cline { 3 - 5 } & $\begin{array}{c}\text { Aumento de } \\
\text { Teor }\end{array}$ & $\begin{array}{c}\text { Aumento de } \\
\text { recuperação em massa }\end{array}$ & $\begin{array}{c}\text { Aumento de recuperação } \\
\text { metalúrgica }\end{array}$ \\
\hline Rougher & Concentrado & $5 \%$ & $25 \%$ & $30 \%$ \\
\hline Cleaner & Concentrado & $2 \%$ & $30 \%$ & $45 \%$ \\
\hline Scavenger & Concentrado & $20 \%$ & $60 \%$ & $25 \%$ \\
\hline
\end{tabular}

Tabela 2: Resultado das variações do contaminante hematita

\section{Conclusões}

Embora a escala dos ensaios seja considerada ainda insuficientes para um tratamento estatístico representativo para o volume de rejeitos gerados, os testes demonstram efeitos na remoção da hematita $\left(\mathrm{Fe}_{2} \mathrm{O}_{3}\right)$ durante a concentração de rejeitos de zinco por meio de concentração magnética de alto campo e compósito magnético de amido modificado em relação aos ensaios em branco. $\mathrm{O}$ incremento de magnetismo causado pelas compósito magnético de amido modificado pode ter auxiliado as partículas finas e ultrafinas de hematita $\left(\mathrm{Fe}_{2} \mathrm{O}_{3}\right)$ a se tornarem mais susceptíveis às forças de campo magnético, resultando em efeitos positivos sobre os teores e recuperações (massa/metalúrgica) do material concentrado magneticamente. A continuidade dos testes e a realização de ensaios utilizando o susceptômetro torna-se imprescindível para que possamos alcançar um nível detalhado de conhecimento e controle sobre os efeitos do magnetismo sobre a concentração magnética de rejeitos de zinco e demais materiais. Futuramente seria interessante a realização de mais ensaios de concentração magnética com outros materiais ferrosos e não ferrosos, sulfetados e não-sulfetados, como por exemplo Fe- $\mathrm{Nb}$ (ferro-nióbio) e $\mathrm{FeTiO}_{3}$ (ilmenita).

\section{Agradecimentos}

Agradecemos ao Prof. Dr. Arthur P. Chaves.

Agradecemos à Ingredion Incorporated pelas amostras de amidos modificados cedidas.

\section{Referências}

1.0 Evans, M. E.; Heller F.; Principles and Applications of Enviromagnetics; Elsevier Science, USA, 2003.

2.0 Bhushan B.; Springer handbook of nanotechnology; Ohio: Springer, 2004.

3.0 Oliveira G.B.; MS Project \& Gestão de Projetos; Pearson Makron Books; São Paulo, 2005.

4.0 Shier; da Costa C.U.; Custos Industriais; Ibpex; Curitiba, 2005.

5.0 Chaves, A.P. Teoria e Prática do Tratamento de Minérios: Flotação o Estado da Arte no Brasil. 1 ed., Signus, 2006;

6.0 Jeong U. et al.; Superparamagnetic colloids: controlled synthesis and applications; Advanced Materials 2007, 33-60.

7.0 Knobel M. et al.; Superparamagnetism and other magnetic features in granular materials: a review on ideal and real systems; J Nanosci Nanotechnol. 2008; 8, 36-57. 